\title{
Research on the Strategy of Backdoor Listing of Chinese Enterprises
}

\author{
Shuliang Liu ${ }^{1, a}$, Huijie Xiao ${ }^{2, b}$ \\ ${ }^{1} \mathrm{NCEPU}(\mathrm{BAO}$ DING) \\ 2 NCEPU (BAO DING) \\ a123IsI@163.com, b18331129376@163.com
}

Key words: Backdoor listing; Listing strategy; IPO

Abstract.In recent years, more and more enterprises are eager to obtain the facility of huge financing or asset merger and acquisition through listing. However not all enterprises can directly take the IPO approach, many enterprises especially medium-sized enterprises have been shut out of the securities market. So there are a lot of non-listed company choose the backdoor listing (RTO) as their way.

From 2013 the backdoor listing has presented a blowout situation, and the successful cases have reached at least 26, the enormous wealth effect of the backdoor listing is popular in the capital market, so it is necessary to regulate the specification of backdoor listing in the current capital market. To study the path and the strategy of the backdoor listing, we can fundamentally master the core of the backdoor listing, and have an in-depth understanding of the backdoor listing.

\section{Introduction}

In China, The initial public offering in the main board and the SME Board shall meet the following conditions: the last 3 fiscal years' net profit are all positive and a total number of more than RMB 30 million; the net operating activities cash flow and operating income in the recent three fiscal years accumulate more than 5000 million and 3 billion respectively, etc.

These conditions above are difficult to achieve for some private enterprises or the enterprises in the initial stage, however the so-called backdoor listing, with respect to the IPO, has such advantages below:

1) Low threshold. Compared to IPO, backdoor listing does not have the requirements for 3 consecutive years of earnings.

2) Short period. Generally IPO needs more than 1 year, if the achievement cannot be calculated continuously, it takes 3 years from the time process of listing. However, the backdoor listing cycle is short, generally from the project start to the success of listing only 6- 9 months.

3) Time interval for refinancing is short. 1 year after first issue and previous funds used up according to provisions before refinancing; however, the listed company can immediately start the financing after the backdoor listing.Backdoor listing can save a lot of time cost, manpower cost and capital cost, so many enterprises prefer to use backdoor listing:

Up to the first half of 2014,soaring shares due to backdoor or recombinant amount to 8 among the first 10 shares of maximum cumulative increase,besides in July 2014, China Securities Regulatory Commission promulgated new regulations defining that the GEM does not allow backdoor listing. So it is necessary to standardize backdoor listing in the capital market, in this way to study the way and strategy of backdoor listing has important practical significance.

\section{The analysis to the strategy of backdoor listing}

M \& A need to obey the overall development strategy, it must be based on the long-term interests of enterprises and the purpose to enhance the overall competitiveness of the enterprise, only under the guidance of the reasonable strategy, the corporate can walk on the right track in the initial stage, the backdoor listing is no exception. 
After determining the shell company, the companies enter the most critical step in the process of mergers and acquisitions, that is, to determine the trading path of backdoor. At present, there are mainly these paths below:

2. 1 Protocol acquisition. Protocol acquisition refers to the equity holders of listed company's transfer their shares to the unlisted companies at a certain price, the transferee company become the controlling shareholder of listed company's, then nonlisted companies use the control of listed companies to reversely acquire the assets of the no listed company thus to achieve backdoor listing.

2. 2 Secondary market purchase. Secondary market purchase refers to the backdoor acquire shares of listed companies through secondary market to gain control of the listed companies, and then the listed company inversely acquire its assets, business, thus realize the backdoor listing, typical cases such as Peking University Founder acquired Yanzhong industrial circulation shares.

2. 3 Purchases of assets through issuing shares. Listed companies issue shares to shareholders or to other companies to buy the assets, then the listed companies have these assets, major shareholders or other companies no longer have these assets and only obtain the shares of listed companies, that is to say, there is not much cash available to listing corporation. The listed company need to issue stock to raise money to buy assets the company needs, such as machinery and equipment.

2. 4 Absorption share type restructuring. Absorbing stock reorganization also known as merger ,the owner of the merger enterprise places net assets of merged corporations as money paid for shares in the shell company, then become a shareholder in the company, after the merger the legal position of the body corporate goals disappear, and will be incorporated into the enterprise merger.

2. 5 Asset replacement. Replacement of assets refers in order to highlight main business, making their asset structure adjusted effective or other reasons the parties involved carry on assets swap. Replace the company's excellent assets in non-listing companies within the group with bad assets in listing companies in order to improve the performance of listed companies, thus realizing the so-called backdoor listing for the subsidiary which has excellent assets.

From 2009 January 1 to 2014 November 20, the total of 62 companies achieve backdoor listing, among them, issue of shares to buy assets is 35, the acquisition agreement has 12,etc.

\section{Table 1}

Composition of backdoor listing

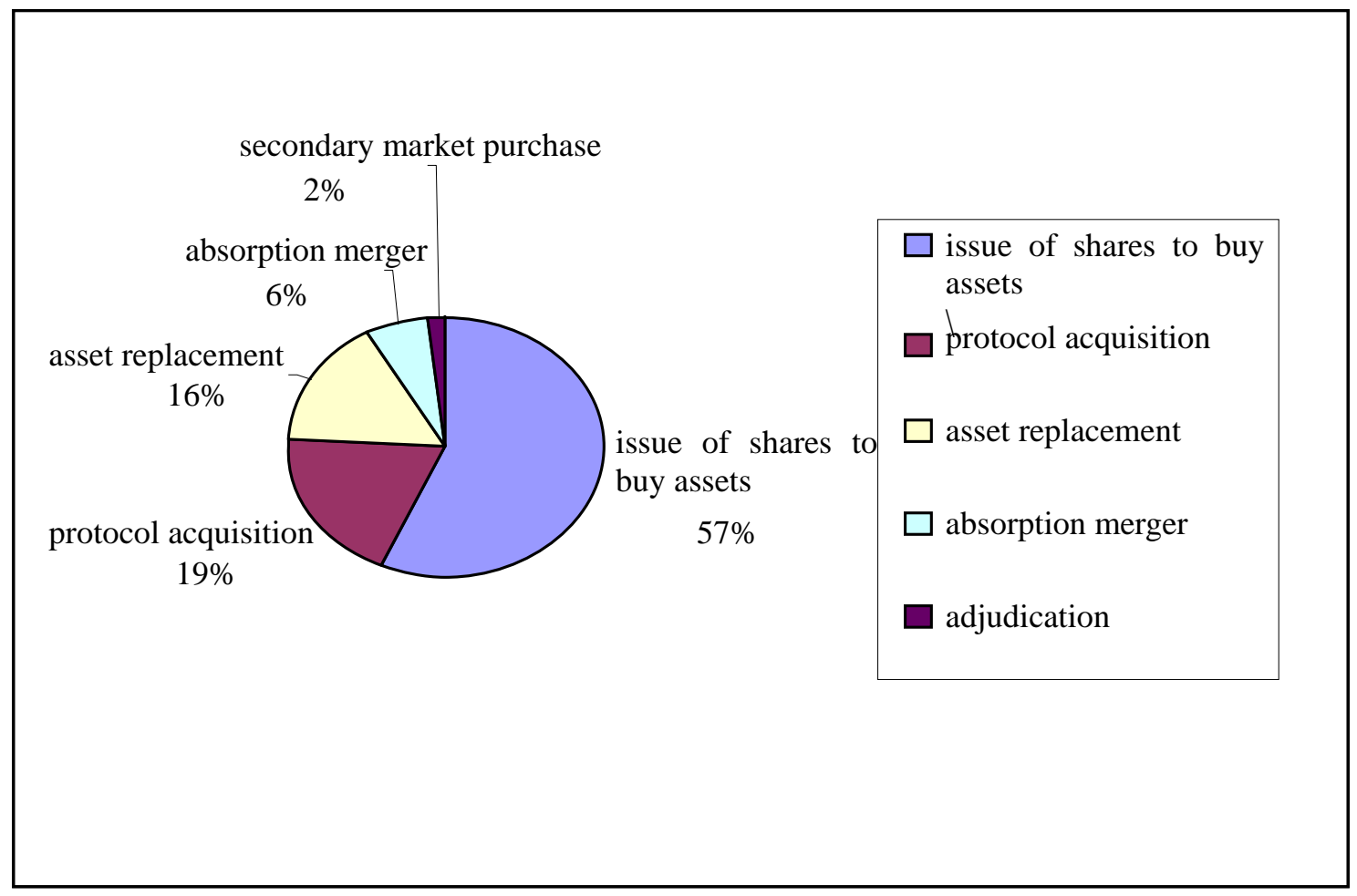


It can be seen above, compared to other backdoor listing way the proportion of issuing shares to buy assets is larger, protocol acquisition ranks secondly, So the Commission should pay attention to these way of listing, making relevant policies and regulations standardized and accommodate to the practice of capital market.

In the whole process of the backdoor listing, the enterprise also faces many uncertain factor, these risks should be taken into account. First, the risk whether the company can effectively control shell company deserve attention. Second, the process of asset restructuring is full of risk, such as whether the injection asset quality is in conformity with the requirements of improving the performance of shell companies and whether the price payment method is appropriate. Third, after backdoor listing due to the failure of reorganization or other reasons, if the competitiveness has not been formed, the performance does not improve or even worse, efficiency is still low, the stock market stock price remains in the doldrums not up to the requirements of company allotment, issuance of new shares and other financing, the backdoor listing strategy ends with failure.

\section{Summary}

Backdoor listing is an effective way to realize the optimization of the resource. Conditional enterprises should choose the most suitable way to achieve backdoor listing based on their own actual situation. Enterprises can use the low threshold of the backdoor listing for capital market financing, in the continuous improvement of innovation ability, the enterprise operator quality and corporate governance structure to promote the backdoor behavior so as to maximize the rational and economic benefits. Indirect listing through the backdoor listing is not the ultimate goal, but the starting point for its further development. In order to obtain the success of the backdoor listing, we need to correctly understand and evaluate the shell company, choose appropriate intermediaries, and conscientiously implement the investigation before buying.

\section{Reference}

[1] L.XV,D.Ding:sumitted to Business Accounting (2008).

[2] W.YONGJIN: sumitted to Time Economy and Trade (2007).

[3] M.LIN JUAN,Y.YAN : sumitted to Friends of Accounting（2008）.

[4] R.GANG YAO: sumitted to Chinese Business(2009). 